\title{
Ragusa Ibla_S. Paolo neighbourhood: Regenerative Cultural Common
}

\author{
Antonella CONTIN, Politecnico di Milano, Italy \\ Patrizia GIORDANO, Fondazione Politecnico di Milano, Italy \\ Valentina GALIULO, Politecnico di Milano, Italy
}

\begin{abstract}
In XX century Italy abandonment is a widespread phenomenon. In the case of S. Paolo - a neighbourhood in the periphery of Ragusa Ibla, a UNESCO site in Sicily -, abandonment is linked to 1) the fragility of its geographical position; 2) the contraction of economic growth; 3) the lack of "modern" urban services. These three factors are interlinked and active as circular causes of the present condition of abandonment of S. Paolo. The paper presents our proposal of intervention working on the three factors together through a two-steps method: first we clarify at different scales the issue (abandonment) and the processes that are producing it; then, we intervene on the three circular and non-linear causes, according to our vision of complexity. Our method also acts on the currently widespread development practices, which could entail the risk of manipulating the identity of a historical place in defining not a collective but only a private space (planning gentrification) and without producing a sustainable project in the long-term spam. We follow a Design Thinkers approach within a Practice of Metropolitan Discipline: every analysis is project oriented and evidence based.
\end{abstract}

\section{Keywords}

UNESCO, Abandonment, Vulnerability, Community Foundation, Metropolitan Cartography

\section{A generative methodology}

\subsection{Background: the three causes of abandonment}

The main urban challenge tackled by the proposal is the abandonment of the S. Paolo neighbourhood - a peripheral district of Ragusa Ibla, a UNESCO site since 2002 (as one of eight late-Baroque towns of the Val di Noto). The abandoned or unused buildings in S. Paolo represent about $65 \%$ compared to the average $42 \%$ of the entire municipal territory (where the population, in 2018, recorded a slight decrease compared to 2017 of $-0.36 \%$, offset by an increase in migration flows +402 , with a general population balance of -247 units). Out of a population resident in Ragusa of 73483 units, in Ibla 1690 reside ${ }^{1}$.

${ }^{1}$ For the statistics, see www.cresme.it and www.bancaditalia.it/pubblicazioni/economie-regionali/2020/2020-0019/index.html 
Abandonments are a widespread phenomenon in the XX century and contemporary Italy (Bonfantini, 2016), already experimented in the same centre of Ragusa Ibla, before the regeneration that began after the town was recognised as a part of the UNESCO site.

From the analysis of statistical data (CRESME; Banca di Italia; Regione Sicilia and ISTAT) we can state that while Ragusa and the historical centre of Ibla are growing in number of employees and tourist attractions, the S. Paolo district is abandoned. It is not a rare phenomenon for the areas of UNESCO sites. All seven UNESCO sites in Sicily have been regenerated, but none of their peripheral neighbourhoods and areas. The phenomenon finds its explanation in the dialectic between cultural good and economic-social good. On the one hand, it highlights the complex patrimonial dimension, which has placed at the centre of attention the cultural value of the historical territories as a quality factor and, therefore, a possible driving force for the development of the different settlement contexts.

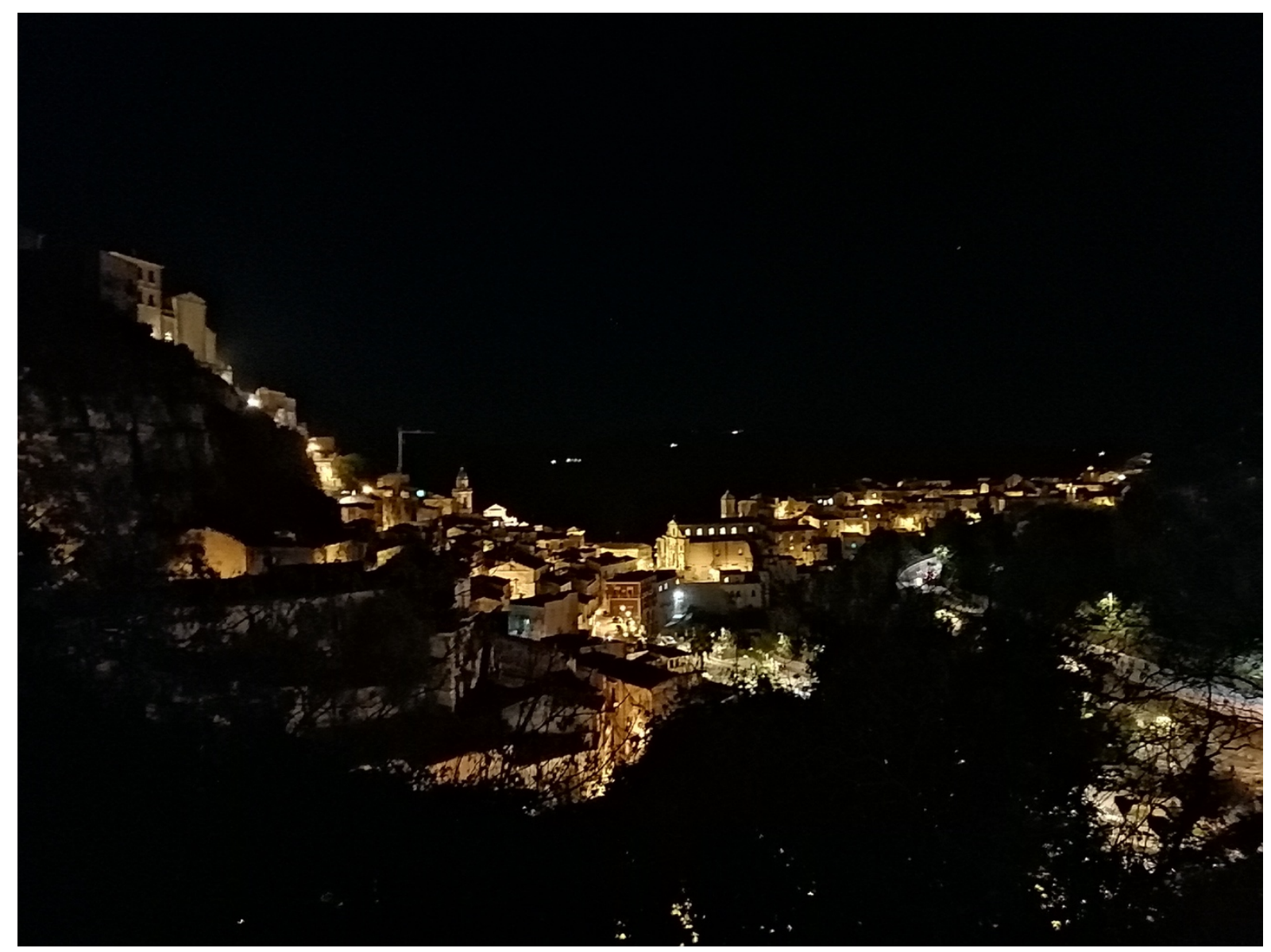

Figure 1. Ragusa - Source: Contin A. 

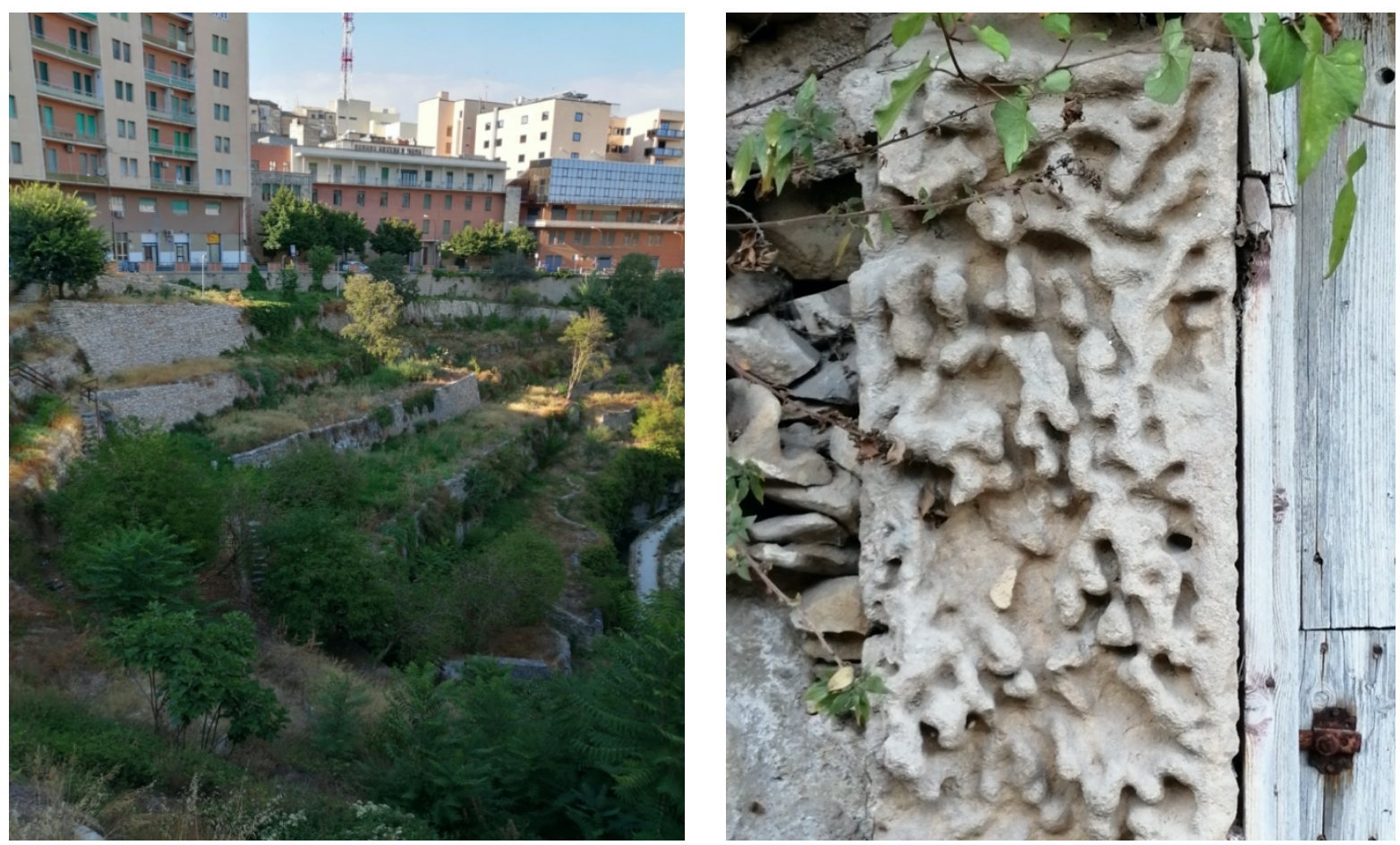

Figure 2. Ragusa S.ta Domenica Valley -- Source: Contin A.

Figure 3. Ragusa Sandstone jamb modified by rain action - Source: Contin A.

On the other hand, there is a vision that recognizes, in those same resources and material presences, a fixed capital waiting to be put to profit. If it still seems right today that the driving force behind the reactivation of historical heritage is tourism, the mechanisms linked to real estate capital prefer to concentrate investments on valuable buildings and consolidated areas. The S. Paolo neighbourhood, however, as a working-class and rural district on the outskirts of Ibla, has been abandoned. The data tell us that there are only 11 activities present (of which ten are for tourists and only one is a kiosk), complicated connections with Ibla and Ragusa, an ageing population. A vital investment decided by the three-year plan of public works in Ragusa ${ }^{2}$ dedicated to mechanical mobility will make the neighbourhood of S. Paolo a new centrality of connection between the historic centre of Ragusa, Ibla and the Valleys (fig.5). However, no plan has been provided for possible future private investment. There is a concern that the urban real-estate market will once again feed a mechanism that distances cities and building products from the real needs of individuals and communities. The "urban real estate market" supported by the drive of ruthless capitalism and the constant search for a global strategy of infinite economic growth - usually prevails over the needs not only of the less affluent segment of the population but also over the needs of the middle class, making ever-larger parts of cities precluded and creating a precise social topography where polarization based on income is increasingly evident.

Therefore, the S. Paolo district abandonment is linked to three factors: the fragility of its geographical position, the contraction of the economic growth (and, specifically, the stagnation of private investments)

${ }^{2}$ Municipality of Ragusa, Triennial Public Works Programme 2019-2020-2021, approved by the Municipal Council Resolution no. 531 of 28 December 2018. 
and the lack of "modern" urban services required by the evolution of the models of social life and use of the city, that define the quality of citizenship in contemporary European society. CRESME data show that funding for public works tendered by the Municipality of Ragusa is growing and that there are significant social or cultural investments both private and linked to the community. The employment rate is increasing: in 2013, out of every 100 inhabitants aged 25-64, 50\% were employed on a national average of 59.8\%; in 2018, unemployment was $27.9 \%$ among the 25-34 year old people, while in the age group of 35 , unemployment was $13.4 \%$, while the gender gap with female non-participation in employment was very high in 2013 (about 40\% against 27\% for men). Ragusa is growing from an economic point of view; but it is not growing on the themes of the conservation of its natural (in the separate waste recollection, for example) and physical heritage. There are only 11 private building permits in Ibla between 2010 and 2019, while many renovation sites were started by the authorities. Nevertheless, also the lack of "modern" urban public, private and collective services, which are required by the evolution of the models of social life and use of the city and define the quality of citizenship in the contemporary European society, motivated the local population to move to other areas of Ragusa.

Since these three factors are interlinked and active as circular causes on the present abandonment condition of S. Paolo, the proposal tackles the challenge with an intervention working on them. We address this challenge since the experience in the same Ragusa Ibla and worldwide shows that in a situation of abandonment the next scenario is very likely to be the loss and dispersion of the cultural heritage and the generation/production of an "artificial" and globalised city, taking place with the intervention of the real estate. Worldwide, if we look at most of the "heart of the city" transformation projects, we see built contexts that are mainly the result of real estate operations, which are characterised by the search for the production-reproduction of capital even at the expense of communities, people and their lives. Built areas, neighbourhoods, cities are becoming financial products, where public good values such as cultural heritage and built and natural capitals, are taken into consideration only in their capacity of maximising profit. Tourism is no an exception. According to the data, in the Sicilian Region the growth of the tourist presence was $2.9 \%$ in 2018 (7.3 \% in 2017) and is now decreasing.

Through a project involving citizens and all agents in the city and territory, our proposal aims to avoid the transformation of S. Paolo into a space that is inaccessible to the low-middle classes and/or the more marginalised groups due to the dizzying increase in rents and the cost of housing. Everywhere in the world, the private speculative real estate activity has transformed the city's historical centres, and also some of its peripheral areas (through temporary "events" that are not integrated into structural and participatory processes and happen to be held). In S.Paolo neighbourhood we aim to counter the negative repercussions and contradictions of the capitalist city's ways of transformation.

\section{The proposed vision}

\subsection{The innovative action}

In recent time Ibla, was transformed in a renowned touristic centre (with high percentage of Michelin Stars restaurants) while its periphery, the S. Paolo neighbourhood, was neither transformed, nor maintained.

In order to face this issue of abandonment and neglection, we intervene on its three main causes defining a whole project based on an integrated approach, from the plan to the assessment of the result of its impact monitoring. The proposal is built on a cross-cutting, non-linear scientific method for integration between a structured system of projects and a participatory process approach. It provides an alternative 
model to the city's mode of production, bringing new life to an urban planning based on the principles of equality, inclusion and civility.

To counteract fragility, our two-steps method propose a first phase of mapping through open-source data (Metropolitan Cartography), remote sensing and OSM of the de facto territorial state of the S. Paolo neighbourhood.

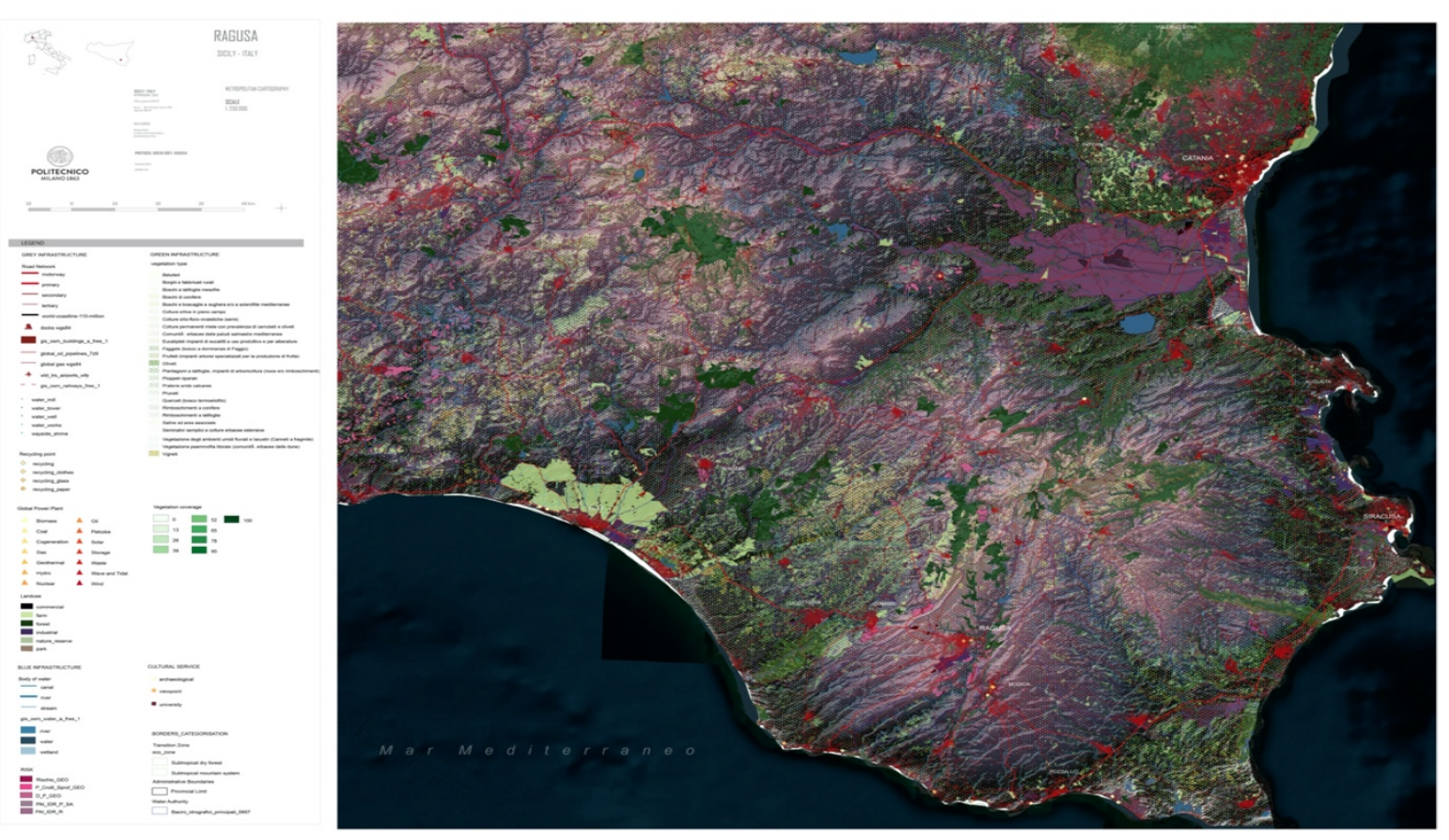

Figure 4. Ragusa Metropolitan Connection Source: Metropolitan Cartography. MSLab. 
We consider necessary to establish a project of local communities dedicated to the growth of learning and capacity in the face of risks. To counteract the economic stagnation, we propose an integral project that can attract investment (in the social, economic and energy spheres) on the heritage of local material and immaterial culture. It is a project of urban regeneration that starts from the integrated accessibility between the two historical centres (Ragusa and Ibla) and the connection of the valleys (S.ta Domenica mainly), and aims to transform the neighbourhood, currently considered peripheral, into a new centrality and to incentivise the return of citizens living there. The project encourages participation and innovation; it acts through alternative proposals of collaborative urban regeneration of the areas, leveraging heritage and culture to regenerate also the socio-economic realm. So, it establishes a Community Foundation ${ }^{3}$ able to keep together places, actors and skills. By reducing the speculative logics based on urban income (one of the factors characterizing today's urban and territorial transformations), we intend to modify the administrative and governance models of the city and the territory based on unbalanced relations between public and private. The aim is to prevent the private sector from guiding the choices of the public sector towards private interests and building waste, as it happened, especially in recent decades. The legacy of that attitude is a phenomenon of exclusion and marginalization of significant social strata not only in some large cities but also, in less economically developed territorial areas, as demonstrated by certain internal European areas. In this way, we intend to innovate the rules of public action in the transformation, restructuring and regeneration of even the most fragile city fabrics, through the implementation of a governance following the metropolitan approach to complexity (Giordano,Contin, 2019) and the projects competitions tool. In fact, we believe that the Governance must deal with the inbetween spaces: looking beyond the traditional definition of borders, aiming at investigating spaces of interaction between local and global forces and powers, challenging fixed administrative borders at different scales (state, region, city). The goal must be not only the balance between the various interests of actors who, although different, must aim to reach an agreement on what is the public good at the local and global scale, but also, a common intention to achieve the agreed objectives. One could think of a public policy model that operates through public competitions that require not only the design and construction of the work but also a more flexible governance strategy between the different levels of

\footnotetext{
${ }^{3}$ A Community Foundation is a non-profit organization, which brings together representatives of a local community (private citizens, institutions, associations, economic and social operators) with the aim of improving the quality of life of the community itself, activating energy and resources and promoting a culture of solidarity and social responsibility. The main peculiarity of this type of foundation is the possibility for a community to invest in its development qualities, activating its own resources in order to carry out projects and interventions for the territory. In addition and as an important subsidiarity tool, a Community Foundation has a strong potential in attracting resources, in the form of donations, since it ensures their investment in local social projects and a careful asset management. The main features of the Community Foundation are: being a private entity with assets; representative of the entire community; autonomous and independent; working for the common good.

To be noted is that the Italian CON IL SUD Foundation promotes the development of Community Foundations in the Southern regions of Italy by supporting their creation and the establishment of their patrimony through mechanisms of grant matching ("doubling of the collection") that stimulate the autonomous fundraising by part of the Community Foundations themselves.
} 
decision-making and management. The instrument of the Community Foundation that we adopt for the regeneration project of the S. Paolo neighbourhood goes in this direction. The city agents gathered together in the Foundation become the shareholders of the project and act in an integrated way to finance and manage it over the time.

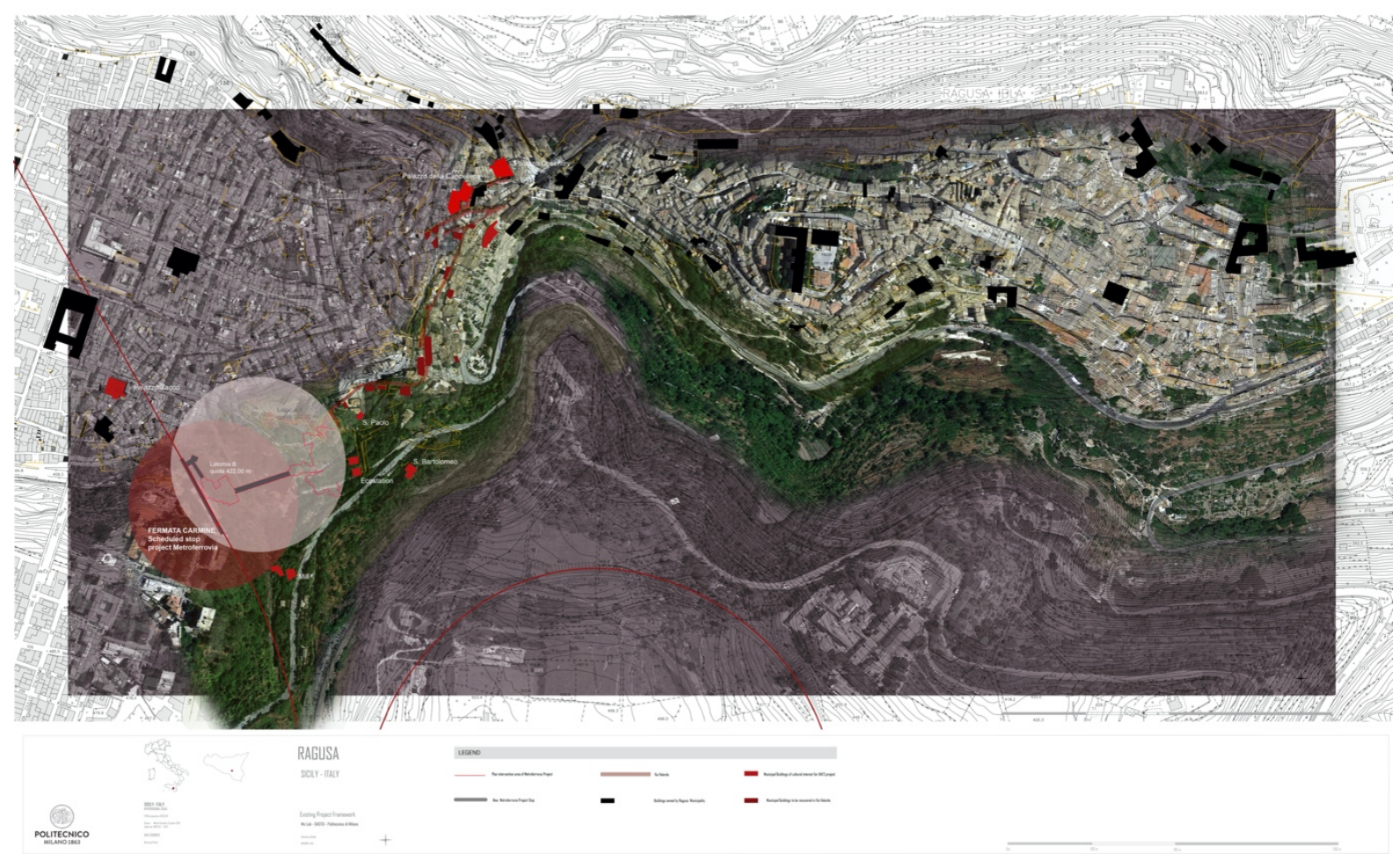

Figure 5. Ragusa. Existing Project Map. Source: Metropolitan Cartography. MSLab

Territorial intelligence is a discipline that aims to advance the sustainable development of various places by strengthening the local communities. This emerging science exploits multidisciplinary knowledge about territories and their dynamics and strengthens the ability of local communities to participate in an equitable and sustainable way in the development of their region. It also endorses spatial information and the sharing of tools, and promotes authority and decisions focused on participation and association $^{4}$ ). Following that approach to counter the lack of "modern" urban services and products (which ensure the right to the city, one of the principles of our methodological approach) necessary for the evolution of new social models and community logic, we define actions for the development of local products able to activate new productive processes related to food, wine and related supplies. The reactivation of the ancient trades is connected to the study of the historical references linked to the territorial intelligences and competences; they bring us back, more than anything else, to the recovery of ancient fruits, to linens and hemp, to sugar cane of Egyptian origin and cultivated in Southern Sicily, to the horticultural production rich in historical varieties. And also to stone and ceramics. The identified solutions to the above-mentioned challenge is therefore in the form of a meta-project that sets the framework and starts implementing a collaborative and integrated regenerative process of the S. Paolo neighbourhood while developing the organizational environment for its linkage to the wider territory.

${ }^{4}$ See the European Commission Sixth Framework Programme (FP6), running in the years 2002-2006. 


\section{The method: innovate to invent something that changes the rules}

\subsection{A widespread gestaltic vision}

In our proposal, S. Paolo neighbourhood within Ragusa Ibla is understood as an Evolutionary city. Our vision and method first clarify the issue and the processes - occurring at different scales - that concurred to determine the issue. Then, the intervention is planned not on the issue directly, but on the main factors that determine it (conceived as circular and non-linear causes, according to our vision of complexity). Our method also acts on the currently widespread development practices, which could entail the risk of manipulate the identity of a historical place in defining not a collective but only a private space (planning gentrification) and without producing a sustainable project in the long-term spam. First, in our interscalar vision the S. Paolo district is located, together with Ibla, within the vast area of the eight late-Baroque towns of the Val di Noto. The valley is the reference system, belonging to Catania metropolitan area (fig. 4, fig. 5). Considered within this dimension, the Project is paradigmatic because it does not consider the territory only as a single and separate case, but as a "spark" that can trigger transformations in the entire metropolitan area. The leap of scale is made necessary by the current moment in the history of the world that we call Anthropocene. Moreover, the Project that intervenes on the three causes of the abandonment of the S. Paolo neighbourhood is an effect of the contemporary historical city centre real estate development approach. We can read the abandonment as an effect of the attraction of the metropolitan areas exerted on the citizens, or of the lack of policies of wealth redistribution. But we can also read the abandonment in terms of geographic urgency: Climate Change; the invasion of vegetation at the expense of agriculture in the valleys; the destructive effect of acidic water on the stones of the houses.

Our project is an innovative practice because it doesn't offer a solution only for a neighbourhood, S. Paolo, or for the city to which it belongs, Ragusa. Our method is about a change in the Gestalt: any local project is planned in connection with the wider areas to which the local scale belongs. Any solution at the local scale recalls endless political choices that give answers at the upper scales to the need for a change of geography, society and politics for the policies of space.

\subsection{Prompts for urban authorities}

According to our method, urban authorities are invited to test innovative solutions to manage demographic trends, to attract relevant economic activities for sustainable urban development from one side, and to counterbalance the effects of demographic decline. This is consistent with the Italian DPS 2013 (Strategia Nazionale per le Aree Interne) which defines specific ways of building the internal areas. Therefore our Project - revolving around three innovative pivotal points that do not act directly on the issue but on its causes - indicates the guide-ideas modifying the negative trends in the territory; the results to be achieved in terms of citizens' quality of life; the actions through which to pursue these objectives.

Without being prescriptive in terms of the types of projects expected, the Ragusa city is invited to consider in particular the following points and issues related to the Dps 2013):

- access to community-based social and health services, incentivizing labour force participation through cultural heritage management and improvement; child/elderly care solutions, improve quality of life through, inter alia, participatory methods with focus on gender and intergenerational dialogue;

- accessibility and sustainability of basic public services;

- reorganisation of existing public infrastructure and services;

- reorganisation of land use and public buildings; 
- development of society 2.0 and 4.0;

- development of the "silver economy";

- strengthening the active labour force by retaining and requalifying the local one and attracting active workers;

- stimulating local entrepreneurship, especially for the new young population.

Reinforcing the capacity of labour market institutions, vocational education, training facilities and lifelong learning. In parallel with larger cities, we strongly think that attention could also be devoted to smaller and medium-sized shrinking cities. If innovative solutions require an urban-rural interface or functional area approach, such as in S. Paolo neighbourhood, it will be possible under this topic to include local administrative units defined as rural (Vallata di S.ta Domenica), according to their degree of urbanization or biodiversity values within a collective project partnership.

Every city needs a tool that helps policymakers to define objectives based on the SDGs and their impact indicators, in order to make decisions that can link the urban metabolism to the regional metabolism.

Our Design Thinkers approach (the designer produces innovation through field experimentation) wants to change the typical rules of the management approach. Our method starts from the problem finding phase, leading to the identification of an issue, and proposes a vision and a solution: what we can do. Then it acts by innovating, and since our action is experimental, we assume the possibility of taking even wrong decisions. We learn through the creation of a balance between existing proposals that are recalibrated as far as the original context to be adapted/translated to the new one is concerned. A budget of mistakes will be the basis for data history as fundamental material for the transferability of the project and its implementation.

\subsection{Case studies on abandonment and innovation}

Since our method aims at designing inter-scalar actions and it is in constant dialogue with practices already adopted and implemented, in the case of $\mathrm{S}$. Paolo neighbourhood we drew inspiration from the rich analysis of case studies relating to places of abandonment presented by the journal Planum in 2016 (Bonfantini, 2016) that can contribute to shape our proposal. In this paragraph we outline therefore some basic and highly shared principles of regeneration and reactivation of cultural heritage (built, natural and intangible) in contexts of abandonment and we also try to highlight how our proposal differs from those experiences. . Three are the needs that our project wants to discuss:

- the need to introduce in these almost completely depopulated contexts an external figure who knows how to recognise a value "other" than the only value of the past and that can be the "spark" for its transformation, because "endogenous cultural factors require the input of exogenous resources";

- the need to consider heritage as an "economic fact" in the broadest sense of the term and not as pure "fixed capital" to be put on income;

- the need to focus not only on the tourism sector as a driver for the valorisation of cultural heritage.

From the analysis of the case studies and, in particular, on some of their actions that have proved to be particularly successful, we can infer possible criteria of orientation among the different experiences. Our project will be based on a model of horizontal accommodation (making reference and taking inspiration from previous projects, such as the Albergo diffuso in Comeglians and S.to Stefano di Sessanio). However, in our case, the challenge will be to attract new temporary inhabitants and not only tourists. For this reason, our users include also students who will be the residents of the neighbourhood for periods 
ranging from one week to several months. The application of the design competition tool - which proposes an implementation of governance and constructive rules, as outlined above in paragraph 2.1 is an innovation proposed by the project. Our project foresees the integration between the S. Paolo district and the natural and agricultural territory of the S.ta Domenica valley. This deeper connection with the valley through the regeneration of ancient paths redesigned for recreation and new experimental agricultural production will offer the tourist - but also the temporary inhabitant - an immersive experience in the material and immaterial heritage of the city and the region (reference project: Case oltre-la-soglia. Metropolitana Milanese Area, Pezzoni, 2018).

For the introduction of the most advanced technological infrastructures (Project: Colletta di Castelbianco), the Municipality of Ragusa has already allocated funds for the development of the Wifi network. In our project, the architecture of public spaces will be powered by this connection that through a mobile application will make the spaces of the city more porous and permeable. The Wifi network will allow the connection between the citizens and the administration, the service providers and the users of the city.

From the experience of the Torri Superiore proposal, our project draws the awareness that the only agrofood activity is not enough to economically support a project of reactivation of abandoned places. The sources of income will have to be differentiated and distributed between food and wine activities, hospitality and training activities.

The case of Castelfalfi shows us the necessity of establishing a relationship between public and private, that our project innovates in aiming to the agreement not between public and private, but between public and common or collective. In our approach, we conceive the commons in the Latin sense of Communis: the possibility of using the good is subordinated to the community work The common good (Community Foundation) is, therefore, a sacred non-public good that cannot be alienated by the public administration. The Community Foundation, capable of holding together actors and knowledge, is also fundamental for addressing the topic of the management of the reactivated spaces, which cannot be entrusted to a single private actor (Project: Montegridolfo). The Community Foundation will also act as an agent for proposals aimed at transforming the administrative regulations that can prevent the sustainable transformation of places; it will happen thanks to the instrument of the project tender, requiring also the presentation of solutions for implementing governance tools. In the end, in our project, the Community Foundation through the work of the social cooperatives included in the partnership, will be instrumental for the inclusion of some marginalised groups living in the city (Project: Riace).

Our project is part of an innovation process related to the implementation of policies for the reactivation and management of cultural heritage, including intangible heritage (Project: Arcevia), introducing actions for skills certification. The training activity is fundamental to respond to the theme of orographic fragility also present in our project area (Project: Craco). That is addressed by proposing a process of mapping through open-source data (Metropolitan Cartography), remote sensing and OSM of the territorial state of the S. Paolo district, necessary to found a project of local communities dedicated to the growth of learning and capacity in the face of risks. A crucial training point is the development of a set of competences related to: knowing the organizational aspect in the space; planning and organising the single actions within the complete restructuring process while putting it into practice, determining the relationship of everybody with the matter to be operated. The certification of these competences must be recognised and constitutes the guarantee of continuity of the training action over time.

Compared to a selection of European Awarded UIA Projects, our project is a case of evolutionary innovation concerning three projects selected by us as reference. The basic idea of "USER - Changes and conflicts in using public spaces \& RE-Block - Reviving high-rise Blocks for cohesive and green 
neighborhoods (urbact.eu/user)" is that the design of urban public spaces and the main objectives of urban planning are challenged by rapid changes in the way cities are used. Also our project, in regenerating the $\mathrm{S}$. Paolo district, will address the theme of public space, conceived as an element (the pocket garden) that is part of the continuity of the green armature of the S.ta Domenica Valley. The urban and architectural project today must be an element of integration between the built and natural capital to achieve the sustainability of the complex system. The themes of the cultural diversity of the ageing population, energy consumption, social exclusion, segregation and social polarisation, the arrival of new inhabitants, is studied at the different scales covering the UNESCO site. Our project acts: through a study of mobility linked to an innovative integrated and multi-dimensional territorial survey that focuses on accessibility and soft mobility; and through the creation of new job opportunities that are generated by a study on territorial intelligence, the subsequent development of their products and the skills they can generate.

Compared to the project "RE BLOCK - Reviving high-rise Blocks for cohesive and green neighbourhoods (urbact.eu/re-block)", the project aligns with the "cultural model" which means the city in continuous evolution and a heritage managed by the whole community of citizens. Our project shares RE-BLOCK's operational objective: "the co-design of actions to produce the city collaboratively, through the combination of specialised actions (decision-makers, technicians) and widespread actions (city actors) in mutual recognition of visions, knowledge, skills". However, this objective is evolved by our project with the introduction of open-source mapping, the Community Foundation and the competences certification.

“URBAN GREEN UP (https://cordis.europa.eu/article/rcn/135393/en)" develops, applies and validates a methodology meant to increase the sustainability of our cities through innovative solutions based on nature. We intervene on the S.ta Domenica Valley by reconnecting it to the city in order to enable the mitigating of the rural-urban contrast that has led to the abandonment of the two components. In the Anthropocene, nature and culture must necessarily collaborate through more porous connections, new land-use patterns (urban-rural linkage) and a renewed relationship with organic and quality agriculture.

\section{Conclusion}

A syntactical transformation is taking place in today's city. Cosmopolitan globalization puts places out of action. Carlo Cattaneo (Cattaneo, 1844) defined the Italian landscape an anonymous regional garden, indicating that the Italian territory, which Kevin Lynch (Lynch, 1960) called "totally built landscape" and "totally visible landscape", is a social work. The identity of a place is a process in which space, time, work and memory are the essential elements for the definition of the identity matrices of geographical, rural, and morpho-typical nature (Contin, 2016). Today, in the discontinuous archipelagos, the new infrastructures articulate and disarticulate that garden. They interrupt the relationships that exist in the territories, and, in addition, they destroy the identity matrix of the agrarian nature. However, it is not cancelled, and the traces of the ancient subdivision of the agriculture fields remain as a trace on the ground, especially as a water line and as an identity image of a characteristic local landscape. It determines a territory whose identity is not static but understood as a dynamic process (Raffestin, 2003).

Alberto Magnaghi (Magnaghi, 2003) profoundly criticizes contemporary design, which reduces the territory to abstract space, to isotropic and inanimate support of economic activities, which generates new poverty due to the degradation of the environment. That, in fact, is what happens when the territory is not understood as individuality and as a set of patrimonial deposits, through which styles of sustainable development are built. Our project proposes a hypothesis of re-appropriation of the territory that starts from local values and strengthens them by creating new relationships between the territory and the mobility networks of settlements and landscapes. This approach aims at establishing a vibrant agro- 
tertiary economy of quality, which starts from the culture of care and the awareness of a shared design around which new relationships are built for the production of lasting and self-sufficient wealth.

We propose a model of a city belonging to the metropolitan region that incorporates agriculture by including new functions and a new idea of public and common space. From a metabolic point of view, growth needs a discontinuity in its structuring, which regulates the logic of dismantling structures that are no longer sustainable. However, against the dissipation of the heritage of the past, the concept of sustainability must be improved. How is the transformation of the territory sustainable? Our answer lies in identifying the knowledge that allows us to know the problem inherent in the transit of scale and the process of metropolisation, which is one of the leading causes of the abandonment of our territories.

\section{References}

Bonfantini G. B.,(2016) Attivare risorse latenti, Roma Milano: Planum Publisher

Cattaneo C., (1844), Notizie naturali e civili su la Lombardia, in VI Congresso degli scienziati italiani, Milano: ip. G. Bernardoni

Contin A., (2016) The narrative structure of the agro-urban metropolitan territory. The metropolis as Hypertext for the history of the XXI century: A network of middle cities as an operational topography, in Petrillo, A., Bellaviti, P., Research for Development Sustainable Urban Development and Globalization New strategies for new challenges- with a focus on the Global SouthBerlin: Springer

Giordano,P., Contin, A., (2019), Training for knowledge to action: tools for the metropolitan architecture discipline, AMPS, Architecture_MPS; Stevens Institute of Technology. New Jersey / New York: 17-19 June, 2019

Magnaghi A., (2003), La rappresentazione identitaria del patrimonio territoriale, in II mondo e i luoghi: geografie delle identità e del cambiamento, a cura di Dematteis G. e Ferlaino F., Istituto di ricerche economico-sociali del Piemonte, Torino, IT

Lynch K., (1960) The image of the city. Cambridge Massachusetts: MIT Press

Pezzoni N., (2018) Case oltre-la-soglia. Metropolitana Milanese Area, in Territorio 85

Raffestin C., (2003), Immagini e identità territoriali, in II mondo e i luoghi: geografia delle identità e processo del cambiamento, a cura di G. Dematteis e F. Ferlaino Ires, Torino

http://www.cresme.it

https://www.bancaditalia.it/pubblicazioni/economie-regionali/2020/2020-0019/index.html

http://www.regione.sicilia.it/bilancio/

https://www.tuttitalia.it/sicilia/25-ragusa/statistiche/

https://www.miur.gov.it/documents/20182/890263/strategia nazionale aree interne.pdf/d10fc11165c0-4acd-b253-63efae626b19

https://www.uia-initiative.eu/en

https://www.comune.ragusa.gov.it/amministrazione/attigenerali/pianotriennale/2019/index.html

https://cordis.europa.eu/article/id/87501-the-smart-approach-to-territorial-intelligence/it 